\title{
A Dynamic-Balancing Testing System Designed for Flexible Rotor
}

\author{
Shibo Zhao $\mathbb{D}$, Xingmin Ren $\mathbb{D}$, Yihao Liu $\mathbb{D}$, Kuan Lu $\mathbb{D}$, Chao Fu $\mathbb{D}$, and Yongfeng Yang
}

Institute of Vibration Engineering, Northwestern Polytechnical University, Xi'an 710072, China

Correspondence should be addressed to Yongfeng Yang; yyf@nwpu.edu.cn

Received 1 August 2021; Revised 12 August 2021; Accepted 17 August 2021; Published 31 August 2021

Academic Editor: Shan Gao

Copyright (c) 2021 Shibo Zhao et al. This is an open access article distributed under the Creative Commons Attribution License, which permits unrestricted use, distribution, and reproduction in any medium, provided the original work is properly cited.

In this paper, a dynamic-balancing testing system is designed. The innovative feature of the testing system is the dynamic balancing of the rotor system with robustness and high balance efficiency which meets the requirements of engineering application. The transient characteristic-based balancing method (TCBM) interface and the influence coefficient method (ICM) interface are designed in the testing system. The TCBM calculates the unbalance by the transient vibration responses while accelerating rotor operating without trail-weight. The ICM calculates the unbalance by the steady-state vibration responses while the rotor system operates with trail-weight and constant speed. The testing system has the functions of monitoring operations synchronously, measuring and recording the required vibration responses, analyzing the dynamic characteristics, and identifying the unbalance parameters. Experiments of the single disc rotor system are carried out, and the maximum deflection of the measuring point has decreased by $73.11 \%$ after balancing by the TCBM interface. The maximum amplitude of the measuring point at $2914 \mathrm{r} / \mathrm{min}$ has decreased by $77.74 \%$ after balancing by ICM interface, while the maximum deflection during the whole operation has decreased by $70.00 \%$. The experiments prove the effectiveness of the testing system, while the testing system has advantages of convenient and intuitive operation, high balance efficiency, and security.

\section{Introduction}

Rotating machinery is widely used in power generation, petrochemical, metallurgy, aerospace, and other fields for its rotating function required in special working conditions. Lu et al. [1] introduced the actual applications of the order reduction methods in solving rotor speed-varying transient problems. Fu et al. [2] analyzed the transient vibrations of an accelerating rotor system under both random and uncertain-but-bounded parameters. In the field of substructures of the rotor system, Xie and Zhu [3] investigated the lubrication characteristics of floating ring bearing under the circumstance of considering multicoupling factors. With the rapid development of high speed and heavy power rotating machinery, detecting and eliminating faults of the rotor system have always been a hot issue in the field of rotating machinery design, research, and development. In the past decades, the research contents of rotor fault detection and elimination include the identification of initial unbalance [4-6], the resonance, flutter and damage monitoring of the blade [7-9], the looseness [10-12], the rotor/stator rubbing [13-16], crack [17-19], and misalignment effects [20-22].

In engineering, the rotor fault of the initial unbalance caused by the process preparation error must be solved. And the rotor system is required to be prebalanced after design and production to reduce the extra vibration response caused by the initial unbalance of the rotor system, which makes the first step of detecting and eliminating faults is the rotor dynamic balancing. The mature dynamic-balancing methods of the rotor system at present are the influence coefficient method (ICM) $[23,24]$ and the modal balancing method (MBM) [25-27]. However, the ICM requires balancing with constant rotational speed. Multiple constant rotational speeds are required to solve the contradiction equations for balancing in a certain range of rotating speed which will definitely reduce the efficiency of the balancing process. The MBM identifies the unbalance parameters by modes of each order and the core principle of the MBM is mode superposition.

Both the above two methods require test runs with trailweight while adding trail-weight to the rotor system may 
lead to two problems. One is that the position of adding trailweight may be sensitive to the main structure of the rotor and may lead to the failure of obtaining the response during the test run with trail-weight. The other is that there is no basis to determine the qualities and directions of the trailweight. Once the acute angle is formed by the direction of adding trail-weight and unbalance azimuth, the vibration response of the rotor system will increase and may cause damage to the main structure of the rotor system.

The mature approach of dynamic balancing without trial weights is to optimize the ICM and the MBM. The parameters that the ICM or the MBM required for calculating unbalance can be obtained through dynamic simulation of the rotor system in this way. And the unbalance of the rotor system can be identified by the ICM or the MBM without trail-weight. For example, Yao et al. [28] proposed a dualobjective optimization method and integrated the proposed method with least-square ICM. Bin et al. [29, 30] investigated the weighted influence coefficient matrix by adding simulated exciting force to the finite element model of the multidisc series shafting rotor system and then identified the unbalance of the rotor system by the ICM without trailweight. Li et al. [31] calculated the dynamic characteristics of the rotor by finite element analysis and identified the unbalance of the four-disc simply supported structure rotor and turbo-shaft engine power turbine rotor by $N$ and $N+2$ plane MBM without trial weights, respectively. Ye et al. [32] introduced dynamic similitude theory and dimensional analysis method for optimizing the ICM and proposed the balancing method without trial weights by analyzing the similitude relationship of the influence coefficient between similarity and prototype rotor system.

Other approaches for balancing the rotor system without trail-weight are based on the dynamic characteristics of the rotor system. Zou et al. [33] estimated unbalance loads of the state space model of the rotor system by using the Kalman filter. Zhao et al. [34] identified the unbalance of the rotor system through transient characteristics of unbalance loads, while the unbalance loads are estimated by vibration response and system characteristics of the rotor system.

Although the methods mentioned above have achieved a good balancing effect in the dynamic-balancing field, the interface of the dynamic-balancing testing system designed and developed in relevant filed is less than other fields such as the interfaces of control systems designed based on excellent control algorithms $[35,36]$, the testing platforms of robot operation designed based on computer vision, virtual reality, and mechanical learning algorithms [37, 38]. The most beneficial function of the dynamic-balancing testing system is field balancing of the rotor system based on the interface integrated by source programs of the specific dynamic-balancing method. Meanwhile, the functions of displaying the measuring signal during operation, analyzing the measuring data in the process of acquisition, are also designed in the testing system, which will simplify the balancing process and improve the balancing efficiency.

In this paper, we proposed a novel method for balancing the rotor system without trail-weight which is named the transient characteristic-based balancing method (TCBM).
The excitation force of the accelerating unbalanced rotor system will be calculated by the transient vibration responses and dynamic load identification technique. And the unbalance parameters are identified by the analysis of the amplitudes and phases of the calculated excitation forces. For simplifying the balancing process and improving the balancing efficiency in experimental research and engineering, the dynamic-balancing testing system is integrated by TCBM. The interface of the ICM is also integrated for demonstrating the accuracy of the unbalance identification by the TCBM and making the testing system more universal.

The basic theories and the balancing processes of the TCBM and ICM are introduced in Sections 2.1 and 2.2, respectively. The composition of the dynamic-balancing testing system, the functions, and the general requirements of the hardware and software modules are introduced, respectively, in Section 3. To verify the balancing effect of the dynamic-balancing testing system, the dynamic-balancing experiments of a single disc rotor system are carried out in Section 4 . The balancing results prove that the dynamicbalancing testing system can be effectively applied to the field of dynamic balancing, while the testing system owns the benefits of concise interfaces and high balancing efficiency and security.

\section{The Basic Methods of the Dynamic-Balancing Testing System}

2.1. The Basic Theory of the TCBM. The TCBM calculates the unbalance parameters of the rotor system by the transient vibration responses and dynamic load identification technique. The TCBM does not require the test run with trailweight and the TCBM is suitable for balancing procedures with the conditions whatever variable rotational speeds or constant rotational speeds. Through the dynamic load identification technique, there is a relationship between the vibration response and the excitation force of the rotor system which can be expressed as

$$
\mathbf{U}(\omega)=\mathbf{H}(\omega) \mathbf{F}(\omega) \longrightarrow \mathbf{F}(\omega)=\left(\mathbf{H}^{T} \mathbf{H}\right)^{-1} \mathbf{H}^{T} \mathbf{U}(\omega),
$$

where $\mathbf{U}(\omega)$ is the Fourier transform of $\mathbf{U}(t), \mathbf{U}(t)$ is the dynamic response vector which represents the output of the system, which is a column vector that contains $L$ elements, and $L$ represents the total number of vibration measurement points. $\mathbf{F}(\omega)$ is the Fourier transform of $\mathbf{F}(t), \mathbf{F}(t)$ is the external force vector which represents the input of the system, $\mathbf{F}(t)$ is a column vector that contains $P$ elements, and $P$ represents the total number of the unbalance excitation force while $L \geq P$ in general. $\mathbf{H}(\omega)$ is the FRF matrix of the system.

Considering the condition of $L=P$, the FRF matrix consists of the modal FRF matrix and the modal shapes matrix which can be described as

$$
\mathbf{H}(\omega)=\boldsymbol{\Phi} \mathbf{H}_{q}(\omega) \boldsymbol{\Phi}^{T}
$$

where $\mathbf{H}_{q}(\omega)=\operatorname{diag}\left[H_{1}(\omega), H_{2}(\omega), \cdots, H_{r}(\omega), \cdots, H_{N}(\omega)\right]$ is the modal FRF matrix and $H_{r}(\omega)$ is the $r$ th modal FRF of the system which can be expressed as 


$$
H_{r}(\omega)=\frac{1}{M_{r}\left(\omega_{r}^{2}-\omega^{2}+2 j \omega \omega_{r} \xi_{r}\right)}, \quad(r=1,2, \ldots, N),
$$

where $M_{r}, \omega_{r}$, and $\xi_{r}$ are the $r$ th modal mass, modal frequency, and modal damping ratio of the system, respectively. $\omega$ is time-varying frequency.

For a nonproportional damping system, the unbalance excitation force can be expressed as

$$
\mathbf{F}(\omega)=\left[\boldsymbol{\Phi}^{*}\left(\boldsymbol{\Phi}^{*}\right)^{T}\right]^{-1} \boldsymbol{\Phi}^{*} \mathbf{M}_{r}\left(\boldsymbol{\omega}_{r}^{2}-\boldsymbol{\omega}^{2}+2 j \boldsymbol{\omega} \boldsymbol{\omega}_{r} \boldsymbol{\xi}_{r}\right)\left[(\boldsymbol{\Phi})^{T} \boldsymbol{\Phi}\right]^{-1}(\boldsymbol{\Phi})^{T} \mathbf{U}(\omega),
$$

where $\mathbf{M}_{r}$ is the diagonal matrix composed of modal mass, $\boldsymbol{\Phi}=\left\{\phi_{1}, \phi_{2}, \cdots, \phi_{r}, \cdots, \phi_{N}\right\}, \Phi *=\left\{\phi *_{1}, \phi *_{2}, \cdots, \phi *_{r}, \cdots, \phi *_{N}\right\}$, $(r=1,2, \cdots, N)$ are the complex modal matrices of the rotor system, and $\phi_{r}, \phi *_{r}$ are the complex modal vectors; the above parameters can be calculated by FEM or modal experiment. $\boldsymbol{\omega}_{r}$ and $\boldsymbol{\xi}_{r}$ are the modal frequency and modal damping ratio, respectively, $\omega_{r}$ and $\boldsymbol{\xi}_{r}$ can be obtained by the Bode diagram of the rotor system, and $\omega$ is the diagonal matrix composed of the time-varying frequency which can be counted by setting the Key Phase Signal (KPS) to the rotor test rig.
In the premise of the radial displacement of the rotor system which can be expressed by the horizontal and the vertical components, the displacement vector in equation (4) can be divided into $x$ and $y$ directions corresponding to the horizontal and the vertical components. Assuming that the rotor system is isotropic and considering the difficulty of obtaining higher-order modes in simulations or experiments of the rotor system, the calculated excitation force vectors can be rewritten by equation (4) which is based on the front $M$ real modal analysis of the rotor system as shown in the following equation:

$$
\left\{\begin{array}{l}
\mathbf{F}_{x}(\omega)=\left[\boldsymbol{\Phi}^{*} \boldsymbol{\Phi}^{* T}\right]^{-1} \boldsymbol{\Phi}^{*} \mathbf{M}^{*}\left(\boldsymbol{\omega}^{* 2}-\boldsymbol{\omega}^{2}+2 j \boldsymbol{\omega} \boldsymbol{\omega}^{*} \xi^{*}\right)\left[\boldsymbol{\Phi}^{T} \boldsymbol{\Phi}\right]^{-1} \boldsymbol{\Phi}^{T}\left[\mathbf{U}_{x}(\omega)\right] \\
\mathbf{F}_{y}(\omega)=\left[\boldsymbol{\Phi}^{*} \boldsymbol{\Phi}^{* T}\right]^{-1} \boldsymbol{\Phi}^{*} \mathbf{M}^{*}\left(\omega^{* 2}-\boldsymbol{\omega}^{2}+2 j \boldsymbol{\omega} \boldsymbol{\omega}^{*} \boldsymbol{\xi}^{*}\right)\left[\boldsymbol{\Phi}^{T} \boldsymbol{\Phi}\right]^{-1} \boldsymbol{\Phi}^{T}\left[\mathbf{U}_{y}(\omega)\right]
\end{array}\right.
$$

where $\mathbf{F}_{x}(\omega)$ and $\mathbf{F}_{y}(\omega)$ represent the calculated excitation force vector of the $x$ and $y$ direction, respectively, $\mathbf{U}_{x}(\omega)$ and $\mathbf{U}_{y}(\omega)$ represent the displacement of the $x$ and $y$ direction, respectively, $\mathbf{U}_{x}(\omega)=\left[x_{1}(\omega), x_{2}(\omega), \cdots, x_{N}(\omega)\right]^{T}, \mathbf{U}_{y}(\omega)=$ $\left[y_{1}(\omega), y_{2}(\omega), \cdots, y_{N}(\omega)\right]^{T},[\boldsymbol{\Phi}]_{N \times M}=\left[\boldsymbol{\phi}_{1}, \boldsymbol{\phi}_{2}, \cdots, \boldsymbol{\phi}_{r}, \cdots, \boldsymbol{\phi}_{M}\right]$, $\left[\boldsymbol{\Phi}^{*}\right]_{N \times M}=\left[\phi *_{1}, \phi *_{2}, \cdots, \phi *_{r}, \cdots, \phi *_{M}\right], \mathbf{M} *=\operatorname{diag}\left[M_{1}, M_{2}, \cdots\right.$, $\left.M_{r}, \cdots, M_{M}\right], \boldsymbol{\omega} *=\operatorname{diag}\left[\omega_{1}, \omega_{2}, \cdots, \omega_{r}, \cdots, \omega_{M}\right], \boldsymbol{\omega}=\operatorname{diag}[\omega, \omega$, $\cdots, \omega]_{M \times M}$, and $\xi *=\operatorname{diag}\left[\xi_{1}, \xi_{2}, \ldots, \xi_{r}, \ldots, \xi_{M}\right]$.

In this way, we can calculate the unbalance excitation forces of the rotor system by the vibration response and the modal parameters of the rotor system.

The excitation force vectors calculated by equation (5) consist of the excitation force of the discs in the time domain which can be expressed as

$$
\left\{\begin{array}{l}
{\left[F_{x}(t)\right]_{Z \times 1}=\left[F_{x 1}(t), F_{x 2}(t), \ldots, F_{x i}(t), \ldots, F_{x Z}(t)\right],} \\
{\left[F_{y}(t)\right]_{Z \times 1}=\left[F_{y 1}(t), F_{y 2}(t), \ldots, F_{y i}(t), \ldots, F_{y Z}(t)\right],}
\end{array}\right.
$$

where $Z$ is the number of discs. The elements of the excitation force vectors contain the unbalance parameters of the discs which can be rewritten as

$$
\left\{\begin{array}{l}
F_{x i}(t)=m_{i} e_{i} A(t) \cos \left[B_{x}(t)+\varphi_{e i}\right] \\
F_{y i}(t)=m_{i} e_{i} A(t) \sin \left[B_{y}(t)+\varphi_{e i}\right]
\end{array}\right.
$$

where $m_{i} e_{i} A(t)$ is the term of amplitude and $m_{i}$ and $e_{i}$ represent mass and eccentricity of $i$ th disc, respectively. $A=\sqrt{\dot{\varphi}^{4}(t)+\alpha^{2}}$, where $\dot{\varphi}(t)$ and $\alpha$ are the angular velocity and acceleration of the disc. $\dot{\varphi}(t)=\dot{\varphi}\left(t_{0}\right)+\alpha t$, and $\dot{\varphi}\left(t_{0}\right)$ is the initial angular velocity. $B_{x}(t)$ and $B_{y}(t)$ are the terms of phases, $B_{x}(t)=\varphi(t)+\psi_{x}(t)$ and $B_{y}(t)=\varphi(t)+\psi_{y}(t) . \varphi(t)$ is the rotational angle of the disc, $\varphi(t)=\varphi\left(t_{0}\right)+\dot{\varphi}(t) t+(1 / 2) \alpha t^{2}$, and $\varphi\left(t_{0}\right)$ is the initial angle of the disc. Generally, we assume $\varphi\left(t_{0}\right)=0$, and $\psi_{x}(t)$ and $\psi_{y}$ $(t)$ can be expressed as

$$
\begin{cases}\psi_{x}(t)=\arctan -\frac{\alpha}{\dot{\varphi}^{2}(t)}, & \psi_{x}(t) \in\left(-\frac{\pi}{2}, 0\right), \\ \psi_{y}(t)=\arctan -\frac{\alpha}{\dot{\varphi}^{2}(t)}, & \psi_{y}(t) \in\left(-\frac{\pi}{2}, 0\right) .\end{cases}
$$

On the premise of the rotor system operating with constant rotational speed, $\dot{\varphi}\left(t_{0}\right)$ is a fixed value, $\alpha=0$, $\varphi(t)=\dot{\varphi}\left(t_{0}\right) t, \quad \psi_{x}(t)=\psi_{y}(t)=0$. For the rotor system operates with variable rotational speeds, $\dot{\varphi}\left(t_{0}\right)$ varies with time and $\alpha$ is approximated as a fixed value; $\dot{\varphi}\left(t_{0}\right)$ and $\alpha$ can be calculated by the KPS.

To conclude, the data of the unbalance force calculated by equation (4) are introduced to equations (7) and (8), and the unbalance parameters (eccentricities and azimuths) of the rotor system can be calculated by equations (7) and (8).

The balancing process of the TCBM is shown in Figure 1.

2.2. The Basic Theory of the ICM. The ICM was proposed by T. P. Goodman in 1964 for the first time. On the premise of 
supposing the system as linear, the residual vibration of the rotor system can be expressed as

$$
\boldsymbol{\delta}=\mathbf{A}_{0}+\boldsymbol{\alpha W}
$$

where $\boldsymbol{\delta}$ is the residual vibration of the rotor system, $\mathbf{A}_{0}$ is the vector which lists the unbalance response of different measuring points while the rotor operates with constant rotational speed, $\boldsymbol{\alpha}$ is the influence coefficient matrix, and $\mathbf{W}$ is the counterweight.

Meanwhile, the element of influence coefficient matrix of the rotor system is defined as

$$
\alpha_{i j}=\frac{\mathbf{A}_{i j}-\mathbf{A}_{i 0}}{\mathbf{T}_{j}}, \quad(i=1,2, \ldots, M ; j=1,2, \ldots, N),
$$

where $\alpha_{i j}$ is the influence coefficient of the $j$ th balancing plane to the $i$ th measuring point. $\mathbf{A}_{i 0}$ is the vibration response of $i$ th measurement points, and $\mathbf{A}_{i j}$ is the vibration response of $i$ th measurement points under the condition of adding the trail-weight $\mathbf{T}_{j}$ to the $j$ th balancing surface. $M=L K$ is the total number of vibration measurement data, $L$ is the total number of vibration measurement points, $K$ is the total number of operating rotational speeds, and $N$ is the total number of balancing surfaces. Generally, $M=N, \boldsymbol{\delta}$ is regarded as the vector whose elements are all zero, and the counterweight of the rotor system can be expressed as

$$
\mathbf{W}=-\boldsymbol{\alpha}^{-1} \mathbf{A}_{0} \text {. }
$$

The single plane influence coefficient method is selected as an example in Figure 2 to illustrate the balancing process of the ICM.

\section{The Design of the Dynamic-Balancing Testing System}

3.1. The Hardware Design of the System. The hardware composition of the testing system is shown in Figure 3, and the KPS was monitored and recorded by eddy current displacement (ECD) sensors or photoelectric (PE) sensors. In the meantime, the KPS needs to be transformed to square wave signals through a signal conditioner for calculating the transient rotational speed. The displacement signals can be monitored and recorded by ECD sensors or General Displacement (GD) sensors; the acceleration signals can be monitored and recorded by acceleration sensors. It should be noted that, in the process of signal measurement, the displacement and acceleration signals do not require transformation, and the rotational speed sensor is required to transform the collected signal into a square wave signal and output the processing square wave signal. All the aboverecorded signals are converted into computers by USB-6351 Data Acquisition (DAQ) system, and the maximum sampling rate of DAQ is $100 k$.

3.1.1. The Design of the Signal Acquisition Channel. The hardware of the testing system can monitor and record 15 channels synchronously during operation, including 8 displacement channels, 1 rotational speed channel, and 6 acceleration channels as shown in Figure 4. It should be noted that all the displacement channels are equipped with two types of sensor interfaces, which can selectively monitor and record the displacement signals by ECD sensors or GD sensors. The rotational speed channel is equipped with the ECD sensor interface and PE sensor interface. The acceleration channels are equipped with the interfaces of acceleration sensors. Therefore, a total of 24 sensor interfaces are designed for the hardware of the testing system, including 2 sensor interfaces for measuring rotational speed, 16 sensor interfaces for measuring vibration responses, and 6 sensor interfaces for measuring acceleration. In addition, the switch, the power interface, and the power grounding are also set to the hardware of the testing system as shown in Figure 5 .

3.1.2. The Introduction of the KPS, Rotational Speed, and Sampling Frequency. In this pattern, the KPS is introduced to obtain the transient rotational speed. It is supposed that the rotor system operates with constant rotational angular acceleration. When the ECD sensor is used to measure the rotational speed, the KPS is triggered by setting the abrupt change of the displacement in the circumferential direction of the shaft. For the PE sensor, the reflective tape set in the circumferential direction of the disc is used for triggering the KPS. In this way, the angular velocity of the rotor between trigger points of two adjacent KPS can be expressed as

$$
\dot{\varphi}(t)=\frac{2 \pi}{t_{k}-t_{k-1}}, \quad(k=1,2, \ldots, N),
$$

where $t_{k}$ is the trigger time point of the $k$ th KPS, $N$ is the total number of the KPS, and $\dot{\varphi}(t)$ is the approximate angular velocity of $t_{k-1}+t_{k} / 2$. And the rotational speed of $t_{k-1}+t_{k} / 2$ can be expressed as

$$
r=\frac{60}{t_{k}-t_{k-1}}, \quad(k=1,2, \ldots, N) .
$$

Equation (13) represents the total number of turns per second. In general, the KPS of the rotor system with constant rotational speed is a series of pulses with equal time intervals. For the rotor system with constant rotational angular acceleration, the time interval of KPS is decreasing regularly in transient motion analysis.

Meanwhile, the KPS plays another role that it can calibrate the rotation angle of the start point of the rotor system during the operation. It should be noted that the reference phase should be set firstly. The setting of the reference phase is very important in engineering which is equivalent to setting a reference frame for operation. We set that the trigger point of the KPS coincides with the positive direction of the $x$-axis and anticlockwise is selected to be positive. In this way, the reference phase is $0^{\circ}$.

The angular velocity at the starting point of the recording data can be expressed as

$$
\dot{\varphi}\left(t_{0}\right)=\frac{2 \pi}{t_{2}-t_{1}}-\ddot{\varphi} \frac{\left(t_{1}+t_{2}\right)}{2},
$$




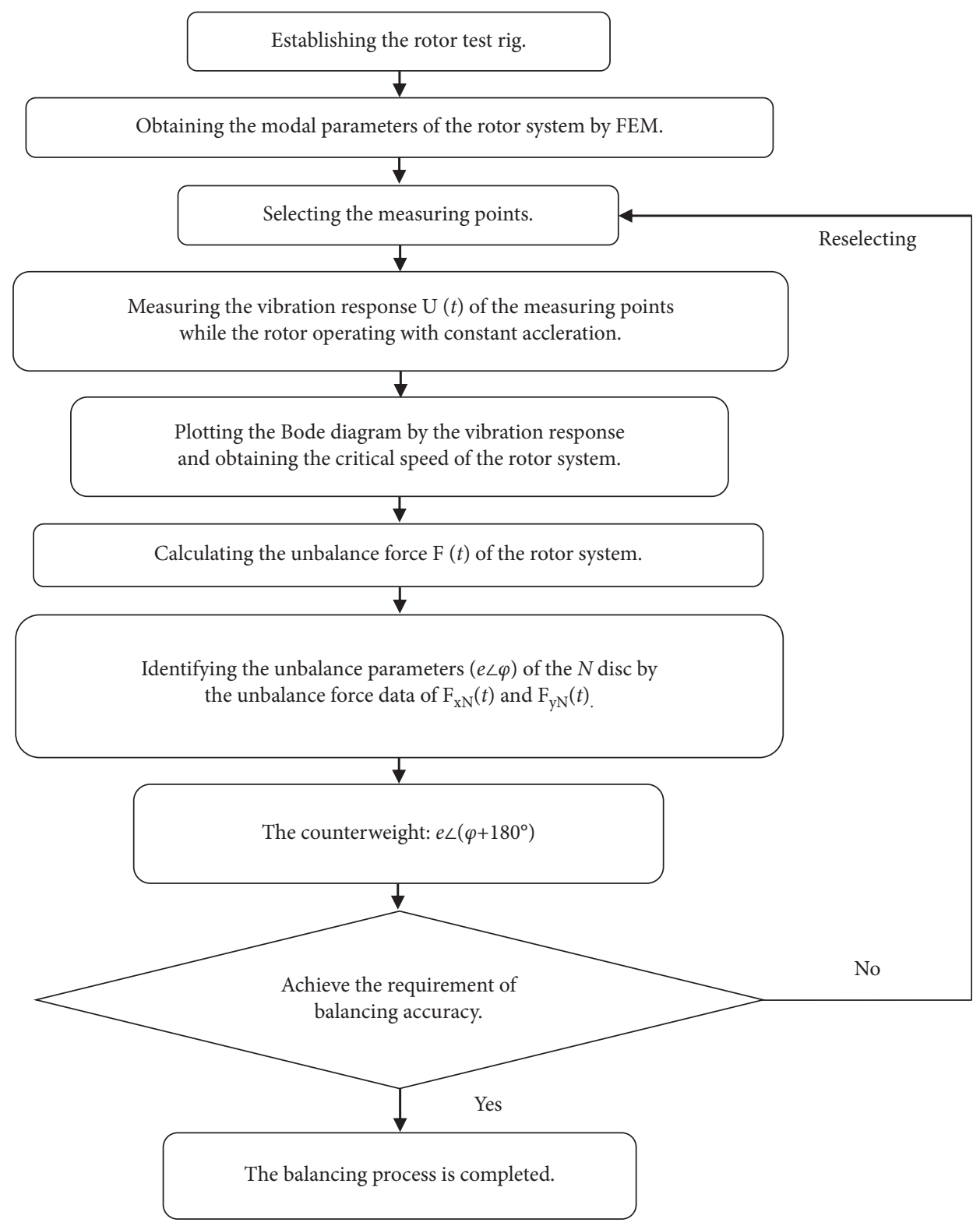

FIgURE 1: The balancing process of the TCBM.

where $t_{0}$ is the starting time, $t_{1}$ and $t_{2}$ are the trigger time points of $1^{\text {st }}$ and $2^{\text {nd }} \mathrm{KPS}$, respectively, and $\ddot{\varphi}$ is the rotational angular acceleration.

The initial phase of the starting point of the recording data can be expressed as

$$
\varphi\left(t_{0}\right)=2 \pi-\frac{2 \pi t_{1}}{t_{2}-t_{1}}+\frac{\ddot{\varphi} t_{1} t_{2}}{2} .
$$

For determining the sampling frequency, it is required that the KPS must be captured at least once in each rotation cycle for the requirements of acquisition and rotational speed monitoring:

$$
\Delta t<\kappa
$$

where $\Delta t$ is the sampling time interval and $\kappa$ is the time width of the KPS.
Equation (16) shows that the sampling time interval is required to be less than the time width of the KPS. Meanwhile, the relationship between the time width of the $k$ th KPS and the width of the KPS in experiments can be described as

$$
\begin{aligned}
\frac{\kappa}{t_{k}-t_{k-1}} & =\frac{\Gamma}{\pi d} \\
& =\frac{\Lambda}{\pi D},
\end{aligned}
$$

where $\Gamma$ is the circumferential width of the abrupt displacement region when the ECD sensor is used for measuring the rotational speed. $\Lambda$ is the circumferential width of the reflective tape for the PE sensor. $d$ is the diameter of the shaft, and $D$ is the diameter of the disc. 


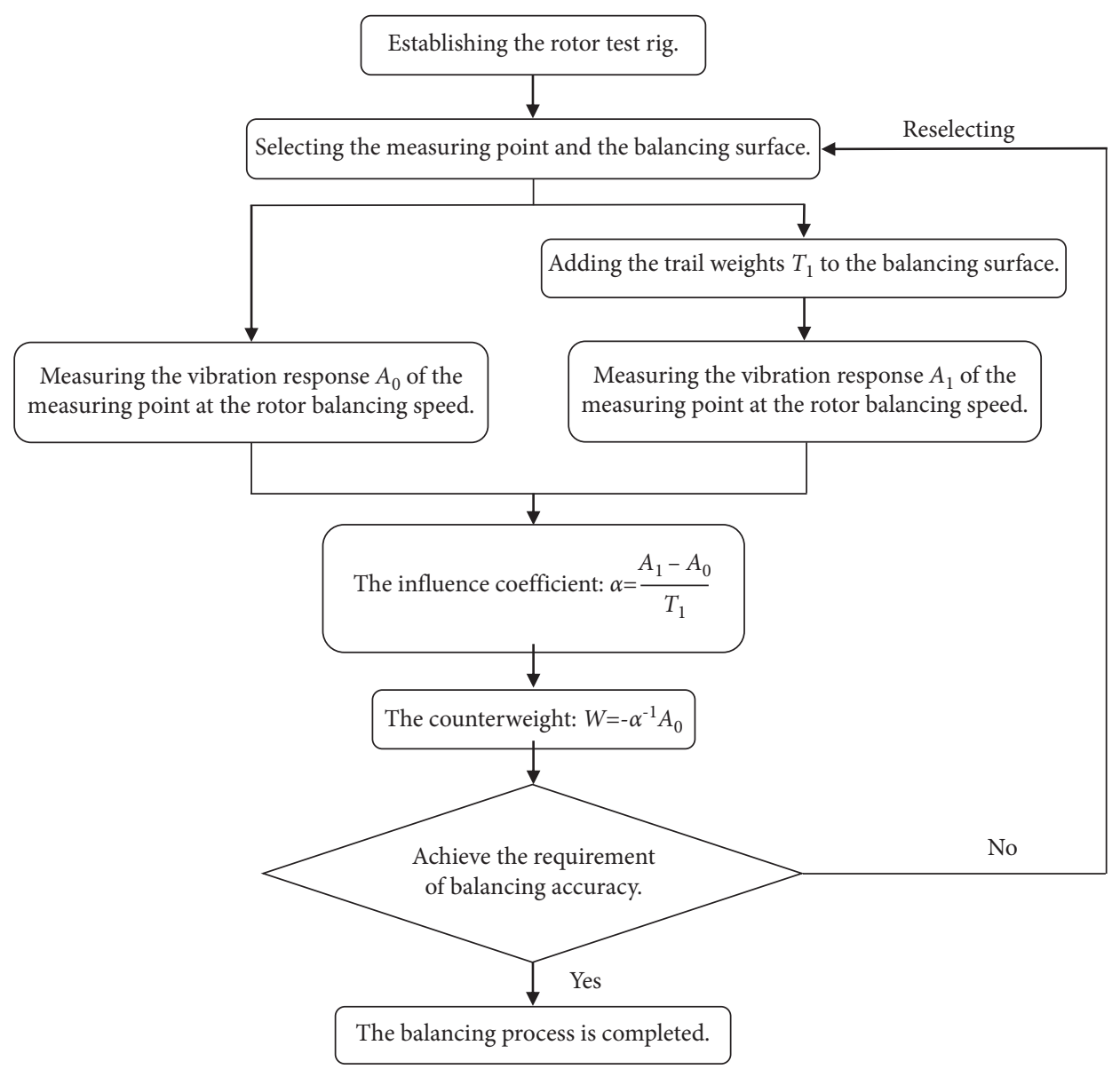

Figure 2: The balancing process of the single plane influence coefficient method.

The sampling frequency of the testing system needs to achieve the following conditions while the rotor system operates with constant rotational angular acceleration:

$$
\left\{\begin{array}{l}
f_{s}=\frac{1}{\Delta t}>\frac{1}{\kappa}=\frac{\pi d}{\Gamma\left(t_{N}-t_{N-1}\right)}, \\
f_{s}=\frac{1}{\Delta t}>\frac{1}{\kappa}=\frac{\pi D}{\Lambda\left(t_{N}-t_{N-1}\right)},
\end{array}\right.
$$

where $f_{s}$ is the sampling frequency of the testing system and $N$ is the last KPS measured by the hardware.

In the meantime, the vibration data acquisition should meet the requirements of the sampling theorem, which is expressed as $f_{s}>2 f_{\max }$ for spectrum analysis and $f_{\max }$ is the frequency corresponding to the maximum rotational speed of the rotor system.

3.2. The Software Design of the Testing System. The software is mainly divided into three parts. One is the preparation before measuring, including parameters set such as sensitivity coefficient, sampling frequency, sampling channel selection, and display. The second part is displaying the measuring signal during operation and analyzing the measuring data in the process of acquisition. The second part can actualize the functions of synchronous display of the KPS, the Bode diagram, spectrum analysis, and the playback of recording data. The last part is the unbalance identification interface which is based on the TCBM and the ICM. According to the unbalance parameters calculated by recording data of the vibration response, the balancing process is completed through adding the counterweights to the balancing surfaces.

The data acquisition interface of testing system software is shown in Figure 6; the buttons of system operation and system maintenance are set in the upper left corner. The system operation buttons own channel setting interface and sensor coefficient setting interface. The drop-down menu of "system maintenance" has the buttons of extracting channel and batch selecting acquisition channels which can realize functions of interface debugging, data transmission fault detection, and daily maintenance.

Meanwhile, a total number of 13 signal windows are displayed in three columns in the data acquisition interface, including 1 KPS display window, 4 measured signal display windows, and the rotational speed-amplitude, rotational speed-phase display windows of the Bode diagram corresponding to the measured signals. The other channels can be displayed by selecting the "channel options" menu at the left end of the interface as shown in Figure 6. The right end of the data acquisition interface displays the amplitude and phase 


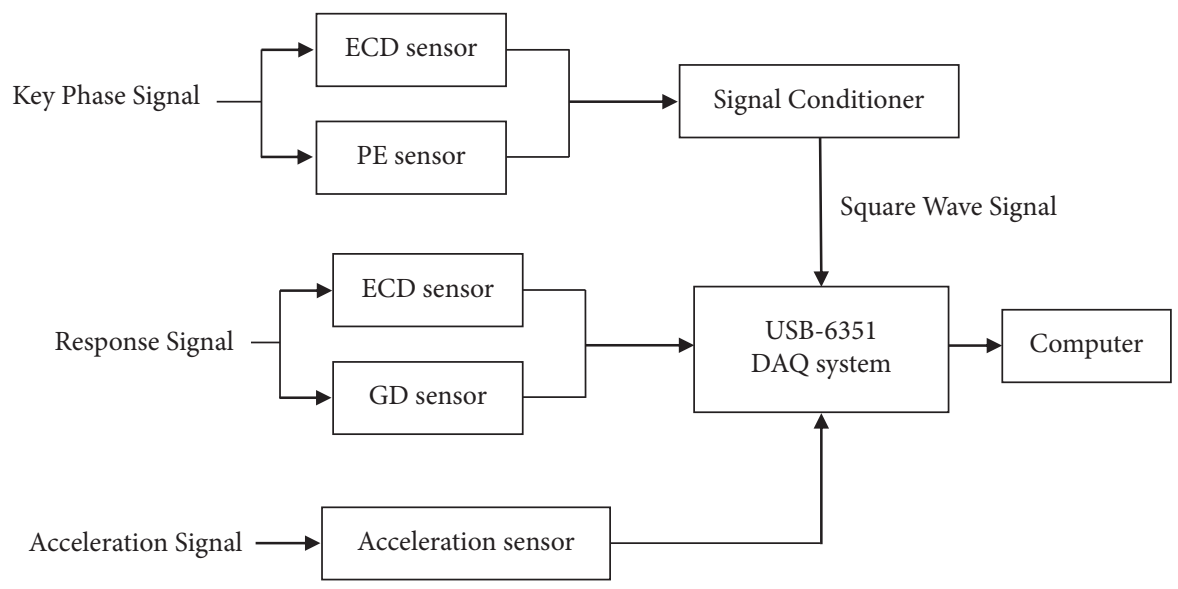

FIgURE 3: The hardware design of the dynamic-balancing testing system.

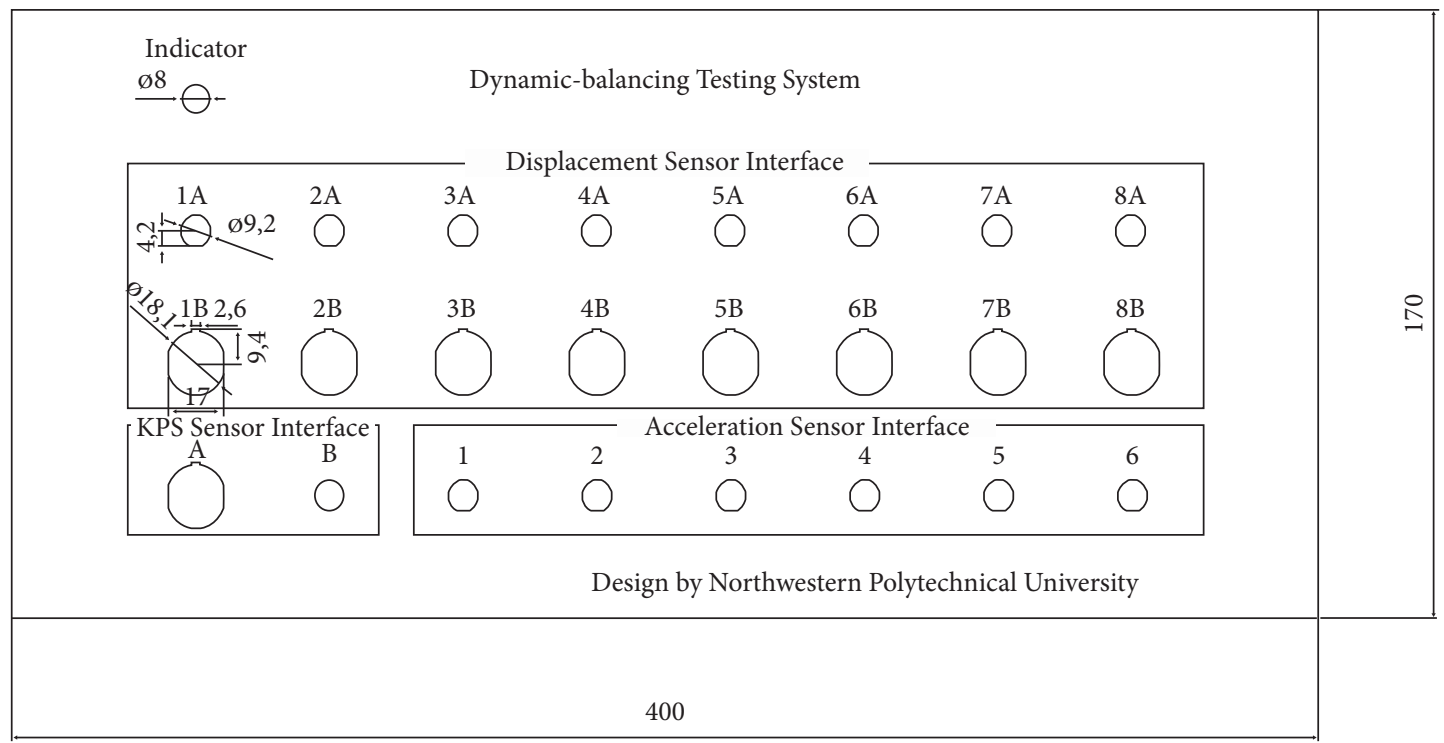

Figure 4: The front layout of the hardware panel.

of the last point in the Bode diagram drawn by the measured signals of each channel. The basic parameters are set below the acquisition interface which includes rotational speed, cycle, and frequency of the rotor test rig, basic setting of sampling. The clear button and the options of adjusting the rotational speed-phase of the Bode diagram are arranged in the lower right corner, which can complete the functions of clearing the plot or phase shift according to the requirements.

3.2.1. The System Operation of the Software. The system operation menu is set in the upper left corner of the software, which has two options in the drop-down menu. One is the acquisition channel setting button and the other is the sensor coefficient setting button. The acquisition channel setting interface is shown in Figure 7. The marks of A and B in the Displacement Sensor part represent the data transmission interface of the ECD sensor and GD sensor, respectively. And a total of 16 displacement channels are set for recording the vibration response. The marks of A and B in the KPS part represent the data transmission interface of the $\mathrm{PE}$ sensor and ECD sensor, respectively. The Acceleration Sensor part owns 6 data transmission interfaces for measuring the acceleration of measuring points.

The sensor coefficient setting interface is shown in Figure 8, which represents the relationship of the sensors' input and output:

$$
\left\{\begin{array}{l}
x_{\text {output }}=\Pi x_{\text {input }}, \\
x_{\text {plot }}=K x_{\text {output }}+b,
\end{array}\right.
$$

where $\Pi$ is the relationship between the input signal and the output signal of the sensor. $x_{\text {plot }}$ is the display signal of the data acquisition interface. $K$ and $b$ are the sensor coefficient, and $x_{\text {plot }}$ should be equal to $x_{\text {input }}$ during operation.

3.2.2. The Bode Diagram, the Recording Data Playback, and Data Processing. For the rotor system, the Bode diagram is 


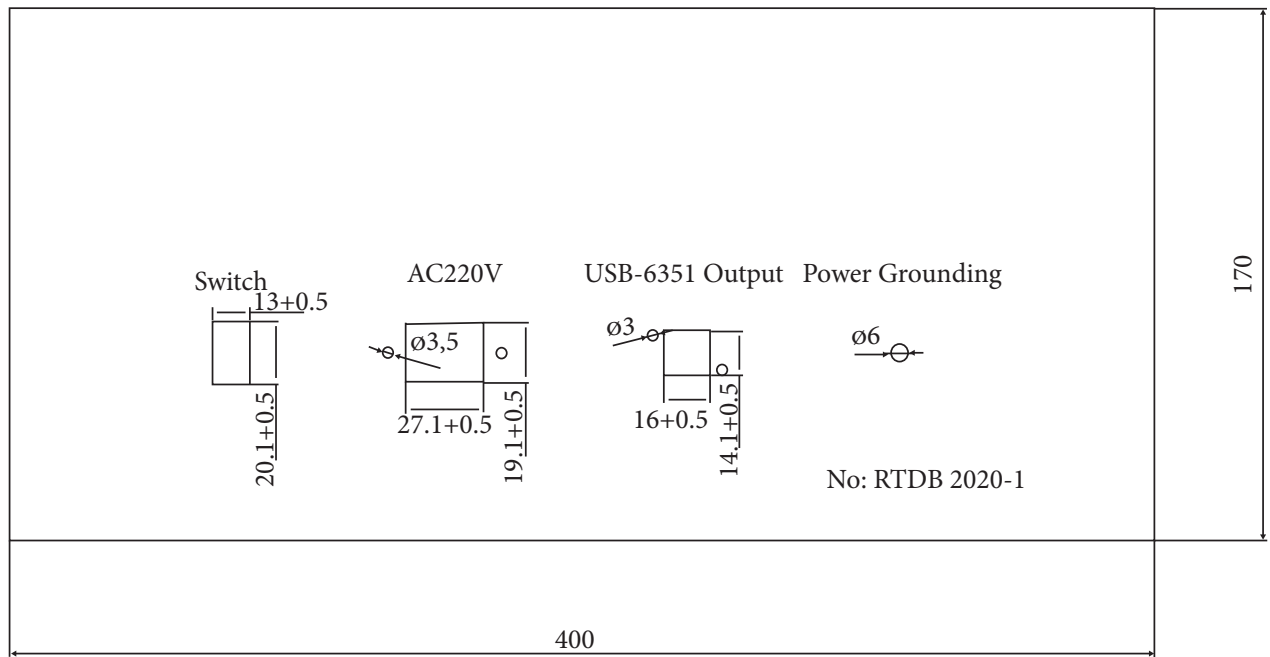

FiguRE 5: The back layout of the hardware panel.

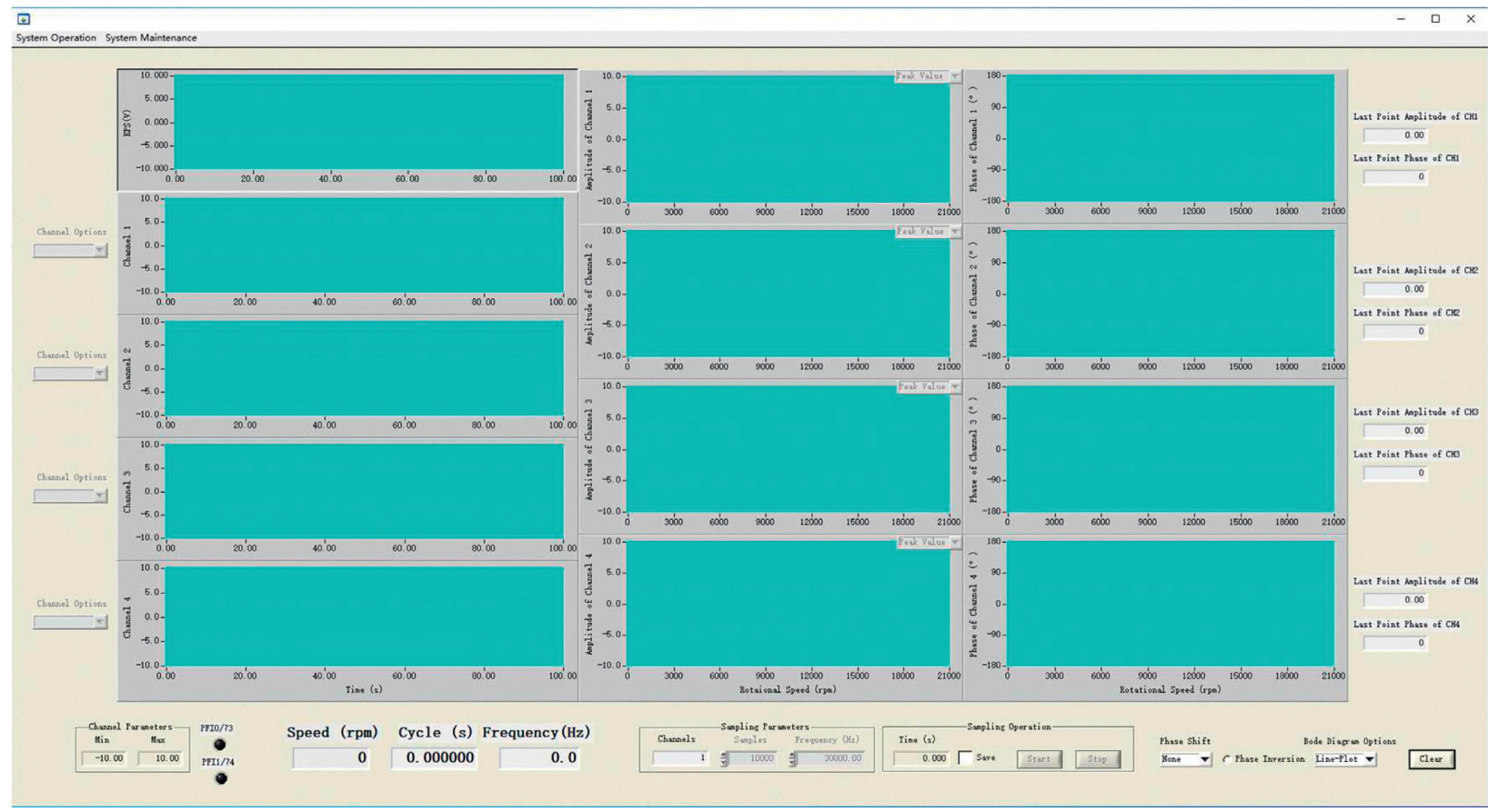

Figure 6: The data acquisition interface of testing system software.

used to illustrate the relationship between the rotational speed and the vibration response of the measuring points. In general, the Bode diagram is plotted in pairs to display the variation of the rotational speed-vibration amplitude and the variation of the rotational speed-vibration phase. In a certain speed range, the amplitude and phase of the fundamental vibration frequency at each constant rotational speed should be obtained for plotting the Bode diagram of the measuring point. The vibration of the measuring point changes periodically with each twirl of the rotor and we assumed that the change of rotational speed in each cycle is approximately 0 . And the amplitude and phase of the fundamental vibration frequency at each corresponding constant rotational speed can be obtained by the Fourier transform to the vibration response of the measuring point in each cycle. Therefore, a series of discrete points of rotational speed-amplitude and rotational speed-phase can be obtained for the rotor system operating with constant angular acceleration. And the Bode diagram can be plotted by curve fitting these discrete points. The $2^{\text {nd }}$ and $3^{\text {rd }}$ column display windows in Figure 6 are the rotational speed-amplitude and rotational speed-phase curves of the Bode diagram, respectively. In addition, the reference phase may be different for engineering in some cases, and the functions of phase shift, phase inversion are set at the bottom right of the data acquisition interface as shown in Figure 6. 


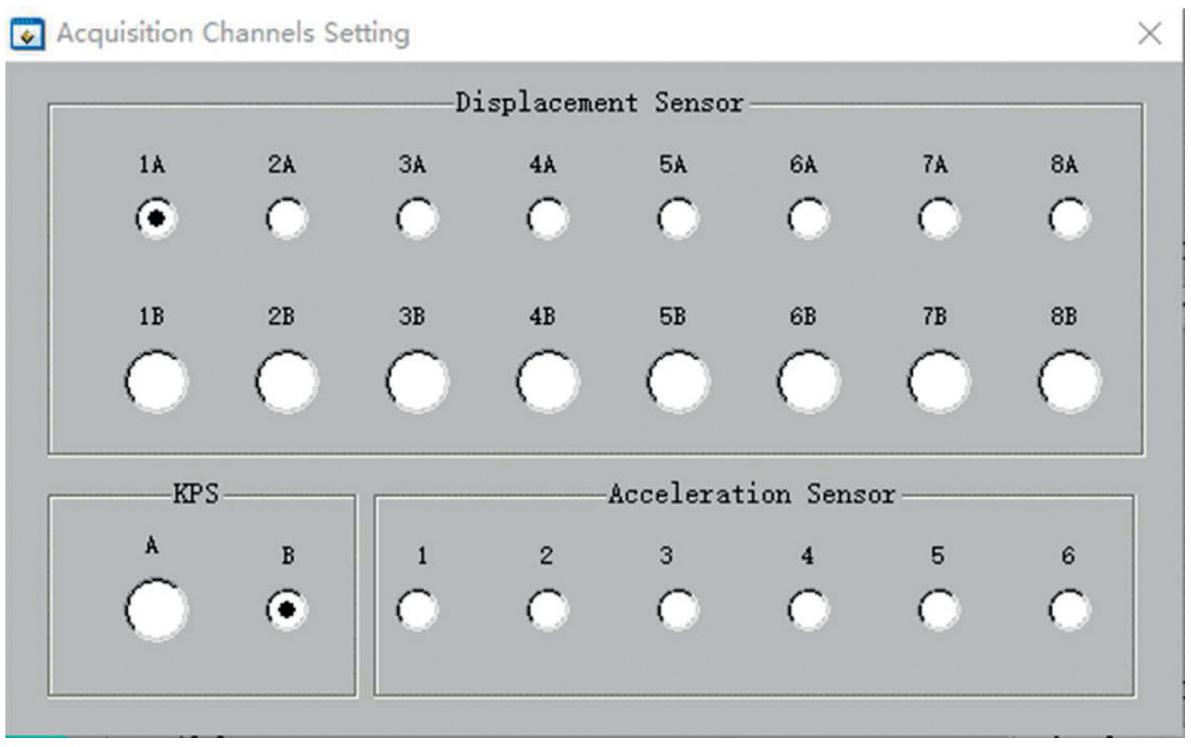

FIgURE 7: The acquisition channel setting interface.

The playback interface is shown in Figure 9, for overall displaying the recording data. The playback windows of the recording data are arranged in the first column, and the display windows of the other column are the spectrum analysis of the recording data. All the windows can zoom and replot aiming to display the details of the recording data. The data reading progress, the basic sampling parameters, and the basic parameters of cursors are set below the acquisition interface.

\subsubsection{The Transient Characteristic-Based Balancing Method} Interface. The transient characteristic-based balancing method interface is shown in Figure 10, which accords with the balancing process of the TCBM shown in Figure 1. The gray buttons in the interface can be clicked in turn based on the reminder for extracting, analyzing, and processing the measuring data of the experiments and calculating the unbalanced parameters. The experimental data of the deterministic channel for analyzing and processing can be extracted by the "File Selection" button and the "Channel Selection" button. After clicking the "Display of the Data" button, the vibration response and the corresponding KPS will be displayed on the image in the interface. The buttons of "KPS Processing" and "Speed-time Curve" are designed for further analyzing, processing the measuring data, and providing necessary parameters for unbalance identification. Meanwhile, the rotational angular velocity-time curve and the rotational acceleration of the selected time interval can be displayed in the interface. Clicking the button named "The Bode Diagram" and the rotational speed-amplitude and rotational speed-phase curve of the Bode Diagram can be plotted on the image. In addition, the critical speed of the rotor system can be obtained by the Bode Diagram. The last part is the process of identifying the unbalance parameters. We can identify the unbalance parameters of the rotor system by clicking the buttons of "The Unbalance Azimuth" and "The Eccentricity."
3.2.4. The Influence Coefficient Method Interface. It should be noted that the interface of the ICM used for the situation of one measuring point and one balancing surface is designed in this paper. And the design concept is the same for the ICM interface to the situation of multiple measuring points and multiple balancing surfaces. The layout of the ICM interface is similar to the TCBM interface. The measuring data of the experiments can be extracted by clicking the gray buttons named "File Selection" and "Channel Selection" as shown in Figure 11. Different from the measuring data under the condition of a constant acceleration extracted for the TCBM interface, the ICM interface requires the measuring data of constant rotational speed. The steadystate response data, the corresponding KPS, and the further processed data will be displayed on the image in the interface after clicking the buttons of "Display of the Data," "KPS Processing," and "Speed-time Curve" in turn. The spectrum analysis of the selected data will be performed after clicking the button of "Spectrum Analysis Interval," and we can obtain the corresponding fundamental frequency, the amplitude, and the phase at the fundamental frequency of the response data of the rotor system with or without trailweight, respectively. The last step is to click the button of "Result" and the counterweight of the rotor system can be obtained in the interface. It can be concluded that the process of the ICM interface used for the situation of one measuring point and one balancing surface requires two interface operations. One is the analysis of the vibration response of the rotor system operating without trail-weight; another is the analysis of the vibration response of the rotor system operating with trail-weight.

\section{The Experimental Tests of the Dynamic- Balancing Testing System}

In this section, the dynamic-balancing experiments of the single disc flexible rotor are carried out which are totally 


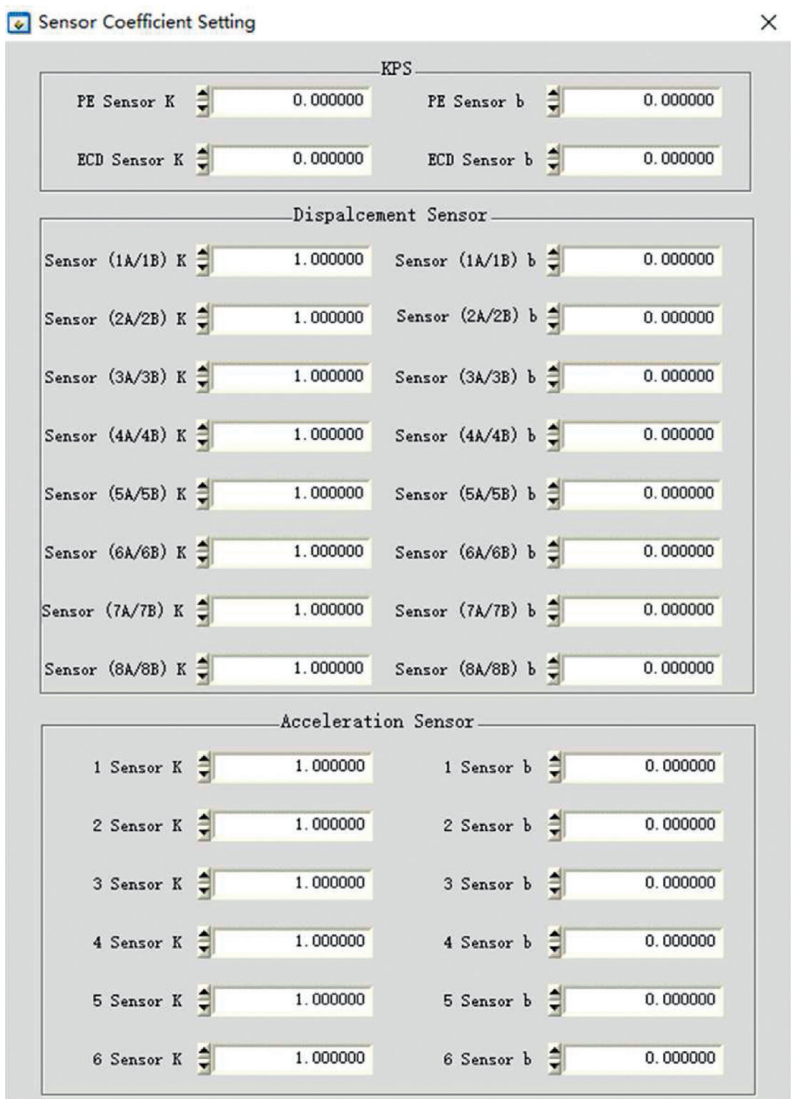

Figure 8: The sensor coefficient setting interface.

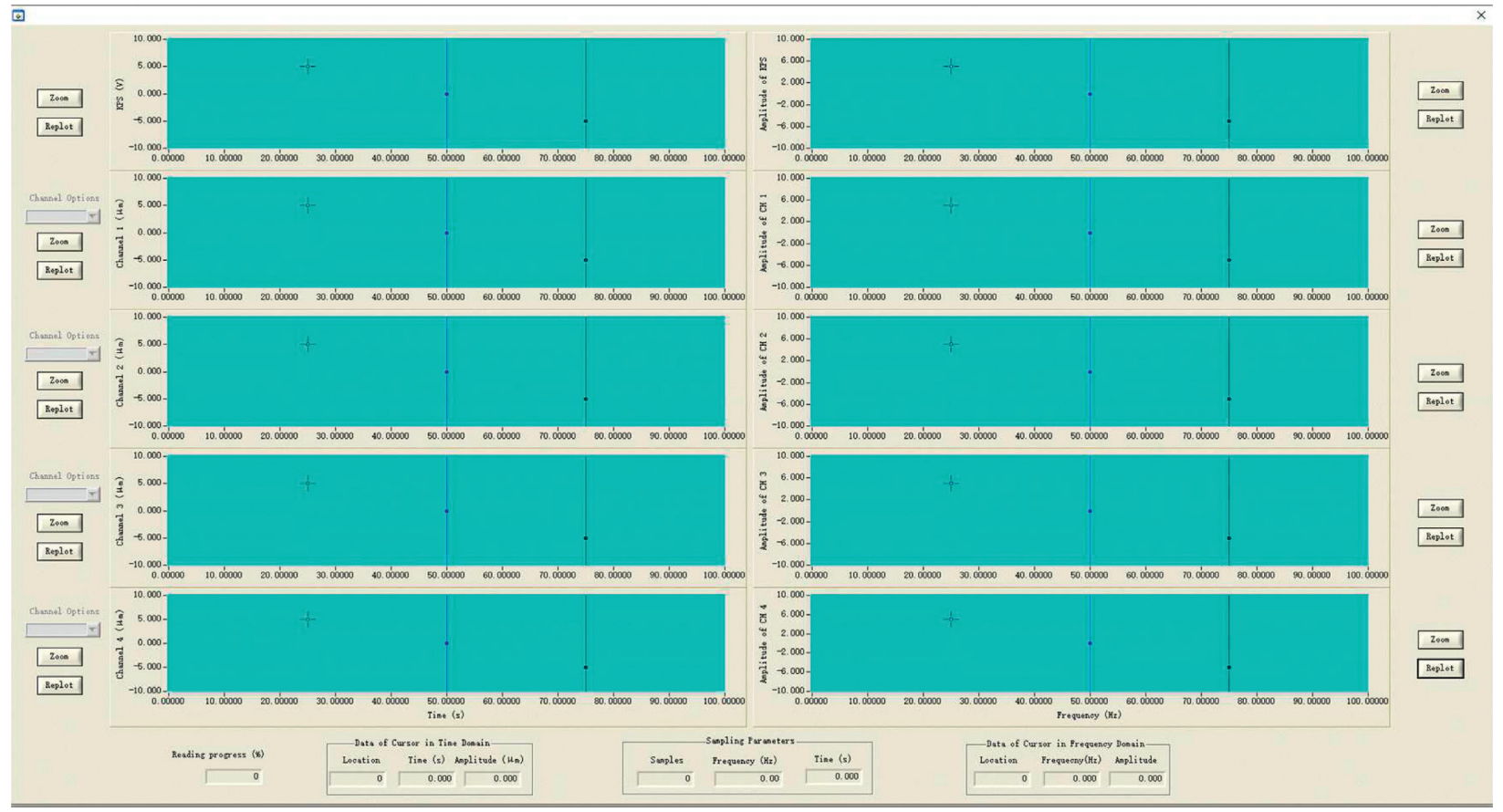

FIGURE 9: The playback interface of recording data. 


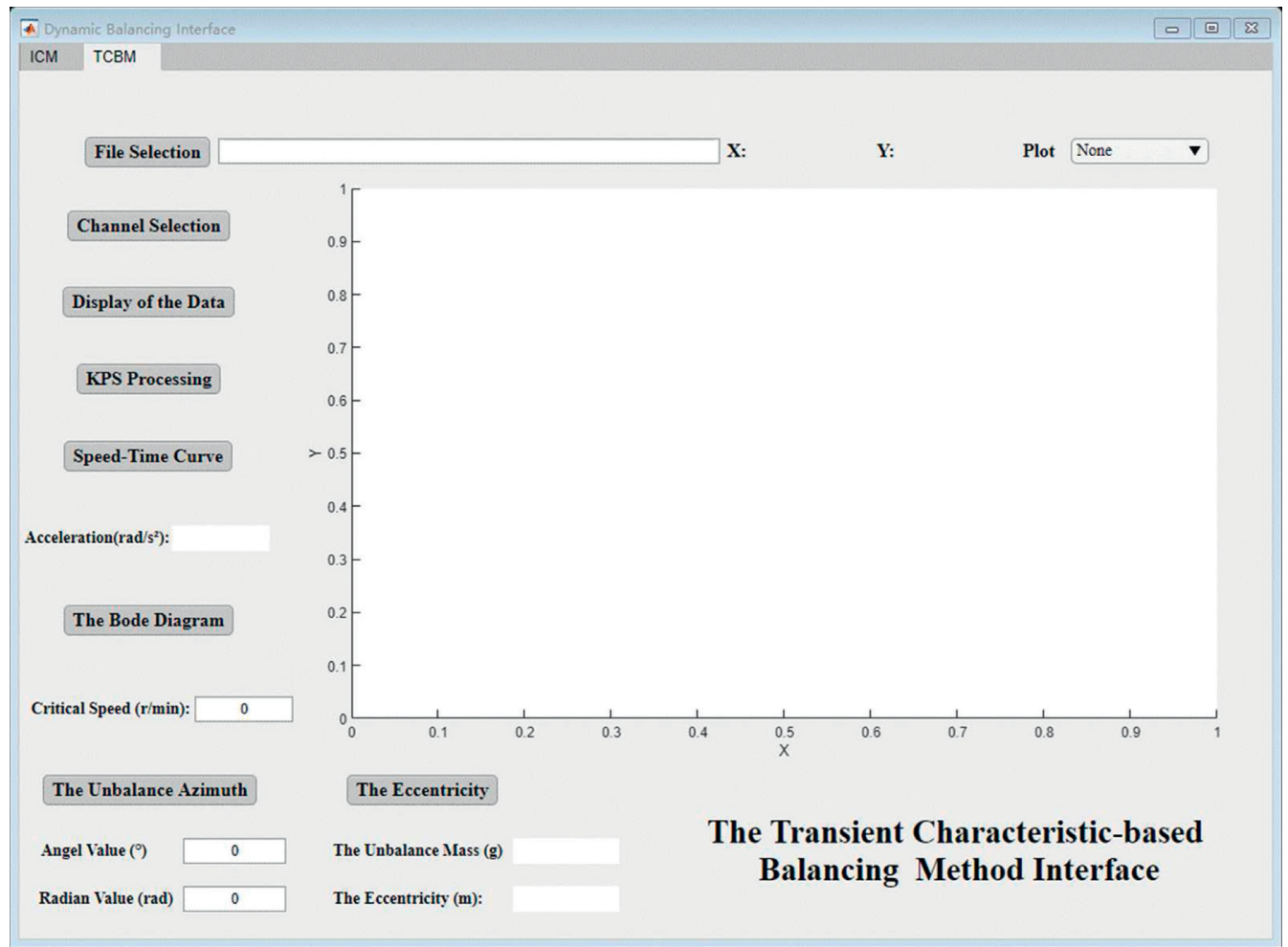

FIgURE 10: The transient characteristic-based balancing method interface.

based on the dynamic-balancing testing system. As shown in Figure 12, the rotor system consists of a disc, a shaft, and two supports. The weight of the disc is $0.48 \mathrm{~kg}$, and there are 16 threaded holes with a radial distance of $0.03 \mathrm{~m}$ on the disc with uniform distribution for adding or removing the counterweights aiming to balance the rotor system. The diameter and the length of the shaft are $0.01 \mathrm{~m}$ and $0.56 \mathrm{~m}$, respectively. Meanwhile, the distance between the left and right supports to the disc are $0.2 \mathrm{~m}$, respectively. The ECD sensors (sensors nos. 1 and 3) are used for measuring the KPS. The ECD sensor (sensor no. 2) is also used for measuring the vibration response of the rotor system, while the displacement of the disc is approximately replaced by the displacement of the shaft section near the disc. The sensor interface of the dynamic-balancing testing system is connected to the corresponding ECD sensor for measuring and recording the experiment data required by the TCBM and the ICM. The TCBM and the ICM interfaces of the testing system are used subsequently for identifying the unbalance parameters according to the recording experimental data, respectively. The KPS and the transient vibration response data of the rotor system before balancing are displayed in Figure 13, while the rotor system operates with constant angular acceleration.

4.1. The Verification of the TCBM Interface in Dynamic Balancing. The unbalance parameters identified by the TCBM interface are shown in Figure 14, while the unbalance results are calculated by the TCBM and the measuring data of the rotor system before balancing shown in Figure 13. The identified result of the unbalance parameters is $7.88 \times 10^{-5} \mathrm{~m} \angle 106.52^{\circ}$, which suggests the counterweight of the disc should be a mass of $1.26 \mathrm{~g}$ placed at $286.52^{\circ}$ with a radial distance of $30 \mathrm{~mm}$ of the disc. Due to a total number of 16 threaded holes being settled on the disc with uniform distribution, the counterweight of $1.26 \mathrm{~g} \angle 286.52^{\circ}$ should be decomposed into counterweights of $0.34 \mathrm{~g}$ to the threaded hole of $\angle 270^{\circ}$ and $0.94 \mathrm{~g}$ to the threaded hole of $\angle 292.5^{\circ}$. Measuring the transient response data of the rotor system with constant angular acceleration after adding the counterweights, the transient vibration responses of the measuring point before and after balancing by the TCBM interface are shown in Figure 15.

As shown in Figure 15, the maximum deflections of the disc before and after balancing are $4.50 \times 10^{-4} \mathrm{~m}$ and $1.21 \times 10^{-4} \mathrm{~m}$, respectively. The maximum deflection of the disc has decreased by $73.11 \%$ after balancing by the TCBM interface, which suggests the effectiveness of the TCBM interface in balancing the single disc rotor system.

4.2. The Verification of the ICM Interface in Dynamic Balancing. Requiring the rotor system operates with lower angular acceleration at first and observing the response signals of the rotor system on the acquisition interface, we can obtain the maximum vibration response of the rotor system while operating near the critical speed. For the single disc rotor system shown in Figure 12, the rotational speed corresponding to the maximum vibration response of the rotor system is $2914 \mathrm{r} / \mathrm{min}$ through observation. On the premise of a constant rotational speed of $2914 \mathrm{r} / \mathrm{min}$, the 


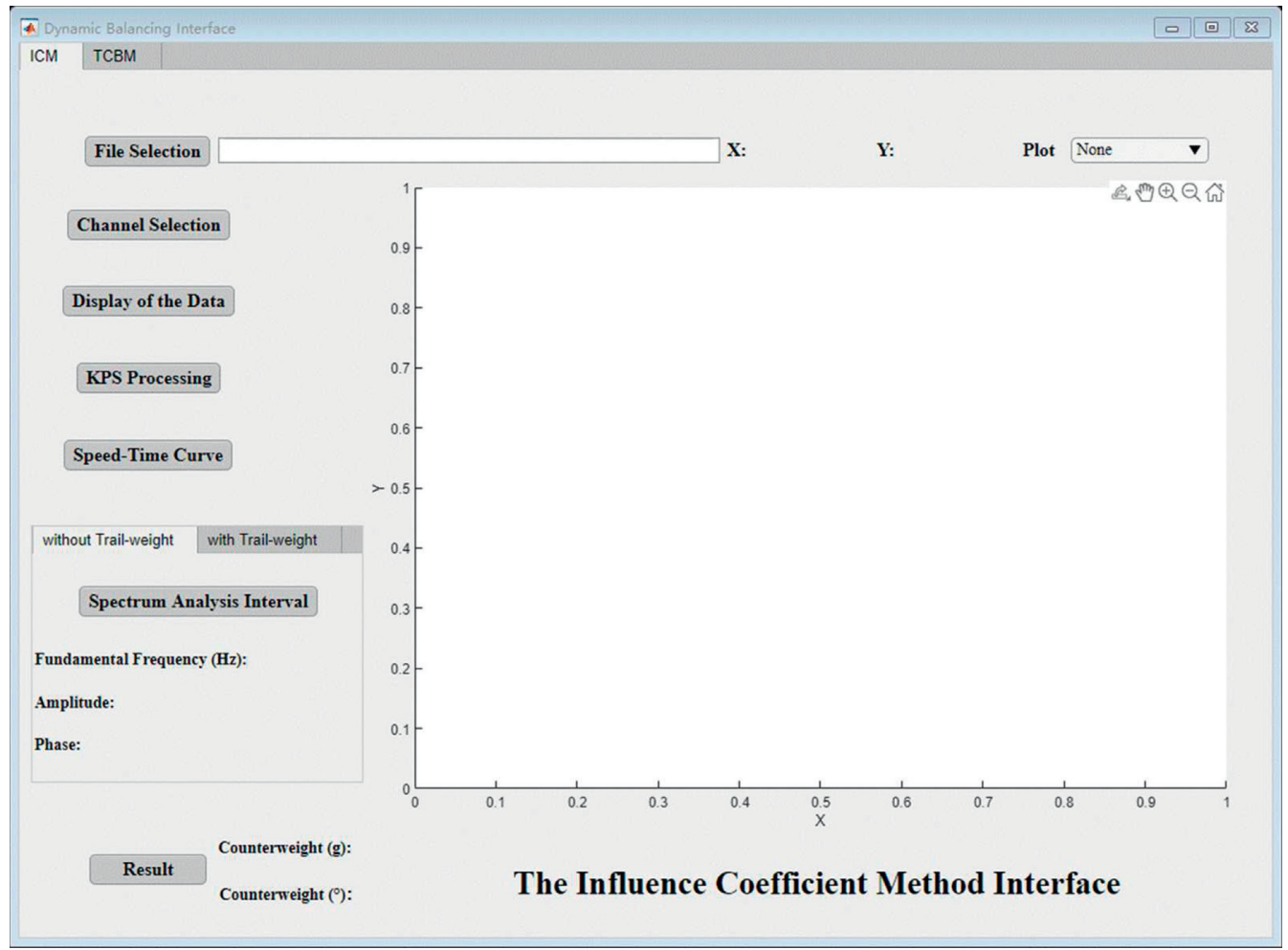

Figure 11: The influence coefficient method interface.

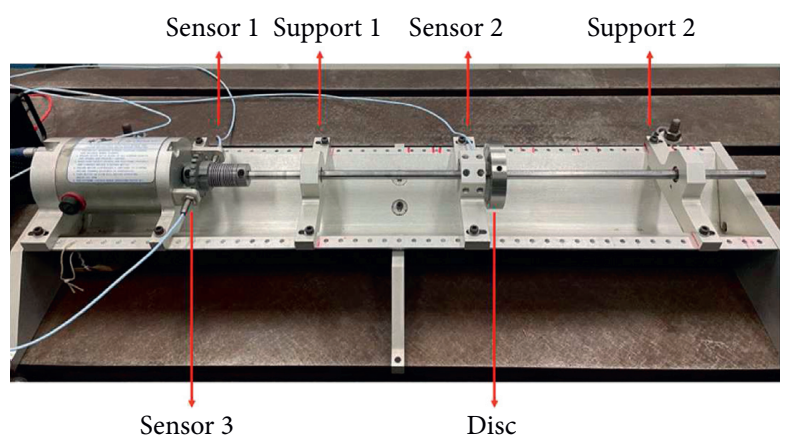

Figure 12: The test rig of the single disc rotor system.

response data of the measuring point in the situation of the rotor system operating without trail-weight and with trailweight are measured and recorded by the ICM interface, respectively. The trail-weight is a mass of $0.72 \mathrm{~g}$ placed at the threaded hole of $0^{\circ}$. As shown in Figure 16, the "without trail-weight" module in the ICM interface is used for analyzing the response data of the rotor system while operating without trail-weight, and the "with trail-weight" module in the ICM interface is used for inputting the parameters of the trail-weight and analyzing the response data of the rotor system while operating with trail-weight. The counterweight calculated by the ICM interface is $1.32 \mathrm{~g} \angle 304.64^{\circ}$, which should be decomposed into counterweights of $0.62 \mathrm{~g}$ to the threaded hole of $\angle 292.5^{\circ}$ and $0.73 \mathrm{~g}$ to the threaded hole of $\angle 315^{\circ}$. Operating with the constant rotational speed of 2914 $\mathrm{r} / \mathrm{min}$ after adding the counterweights to the disc, the comparison of the steady-state vibration responses before and after balancing by the ICM interface is shown in Figure 17. Figure 18 is the comparison of the experimental transient deflections before and after balancing by the ICM interface.

It can be summarized from Figures 17 and 18 that the steady-state vibration amplitudes of $2914 \mathrm{r} / \mathrm{min}$ and transient deflections are all significantly decreased after balancing by the ICM interface. The maximum vibration amplitudes of the rotor system at $2914 \mathrm{r} / \mathrm{min}$ before and after balancing by ICM interface are $4.47 \times 10^{-4} \mathrm{~m}$ and $9.95 \times 10^{-5} \mathrm{~m}$, which have decreased by $77.74 \%$. In Figure 18 , the maximum deflection has decreased by $70.00 \%$ while the maximum deflections of the disc before and after balancing 
(9)

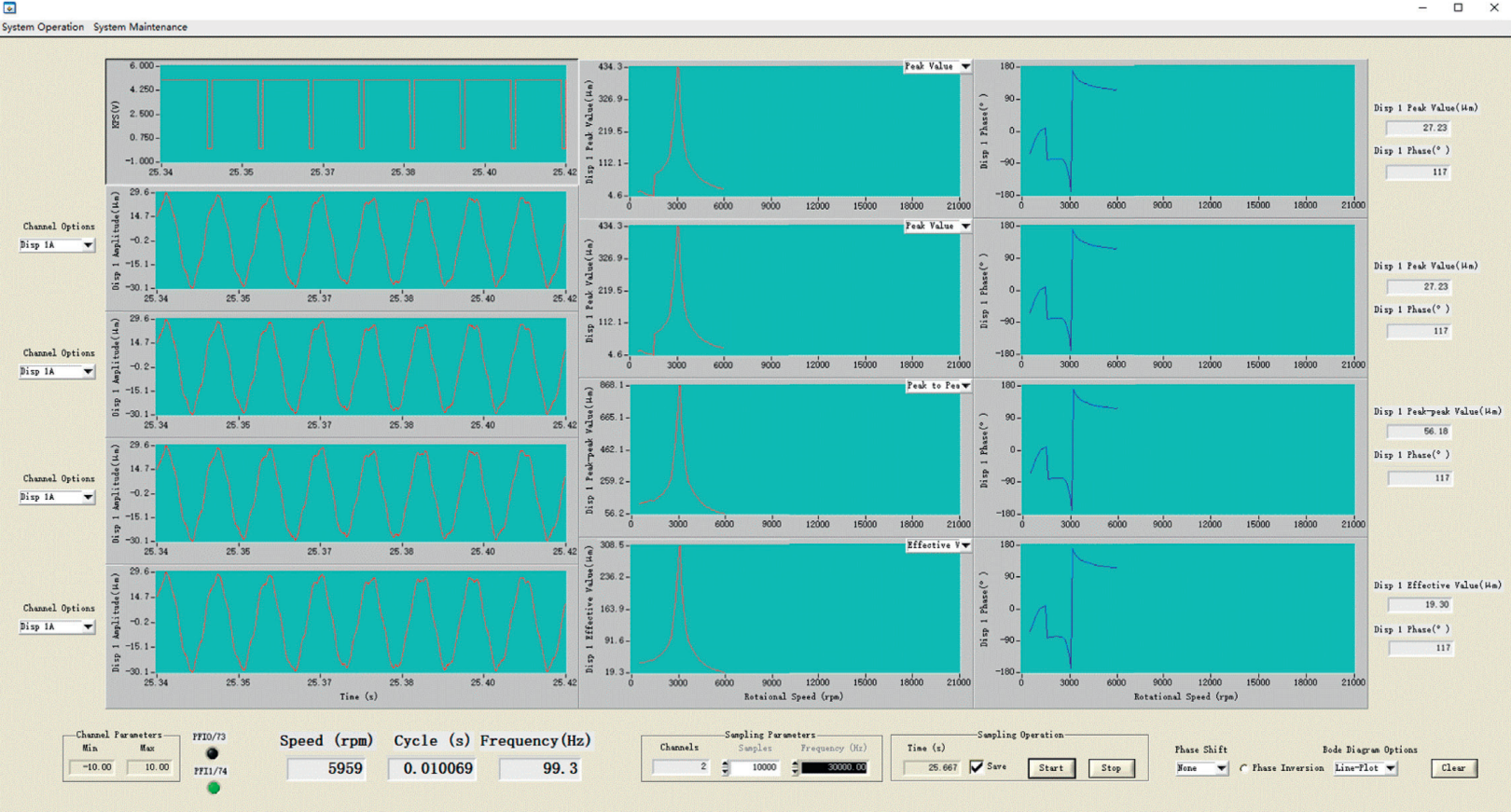

FIgURE 13: The measuring data of the rotor system before balancing (operating with constant angular acceleration).

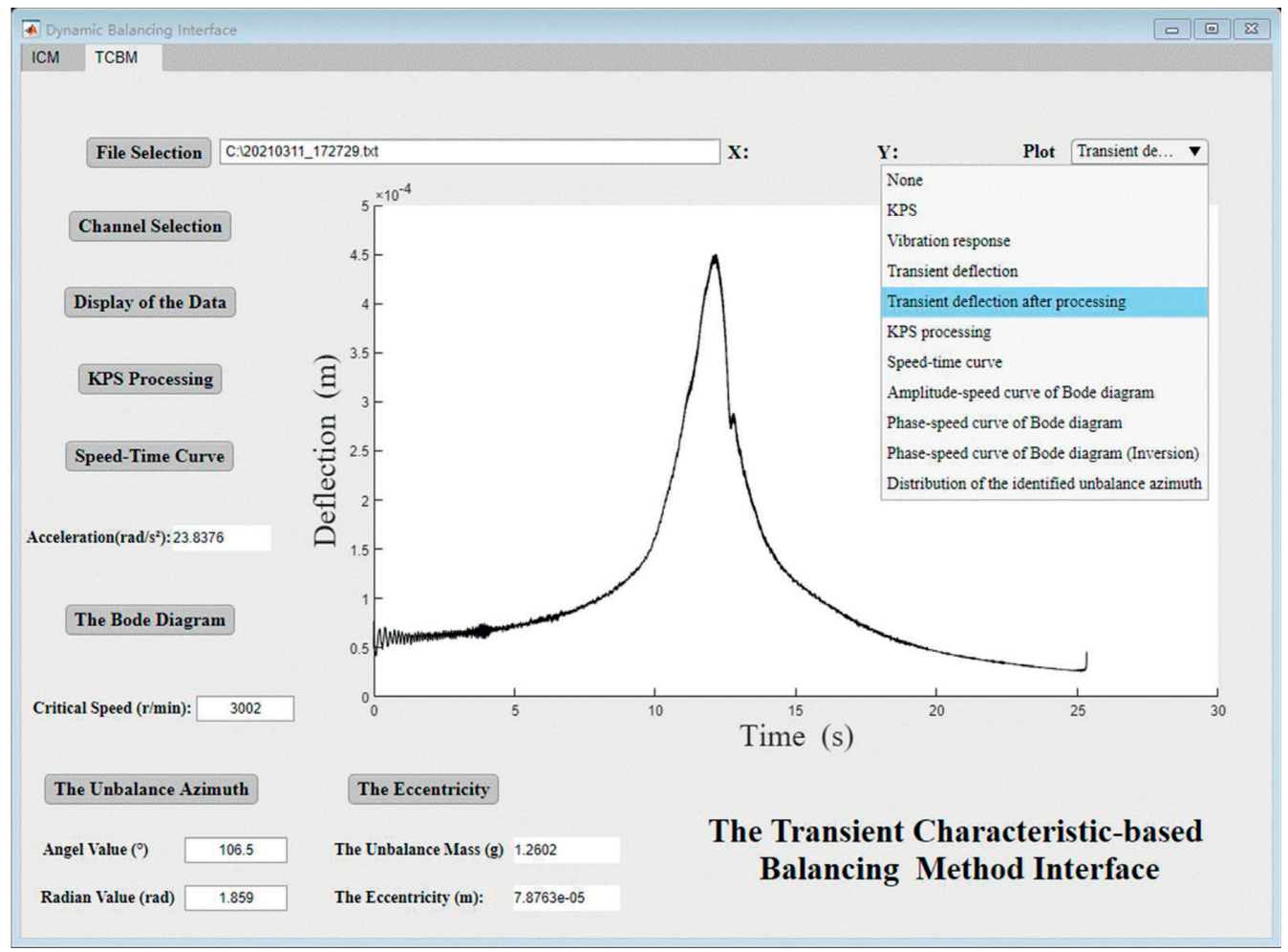

FIgURE 14: The results of the unbalance parameters identified by the TCBM interface.

are $4.50 \times 10^{-4} \mathrm{~m}$ and $1.35 \times 10^{-4} \mathrm{~m}$, respectively. These results prove that the ICM interface has good performance for balancing the single disc rotor system.

In conclusion, the experiments in this section show that the dynamic-balancing testing system succeeds in balancing the single disc rotor system by both the TCBM interface and the ICM interface. The TCBM interface identifies the unbalance parameters by the transient vibration response while the rotor system operates with constant angular acceleration, and the maximum deflection of the measuring points has decreased by 


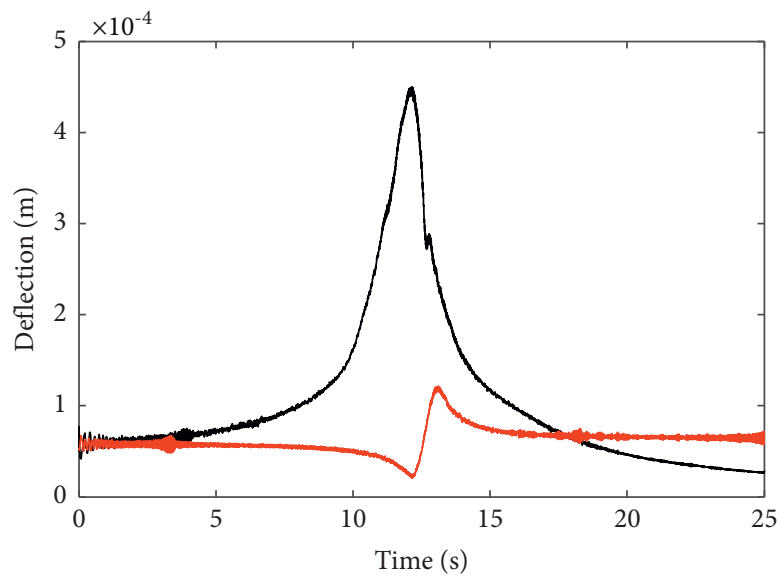

Before balancing

After balancing

Figure 15: The transient deflection of the measuring point before and after balancing by the TCBM interface.
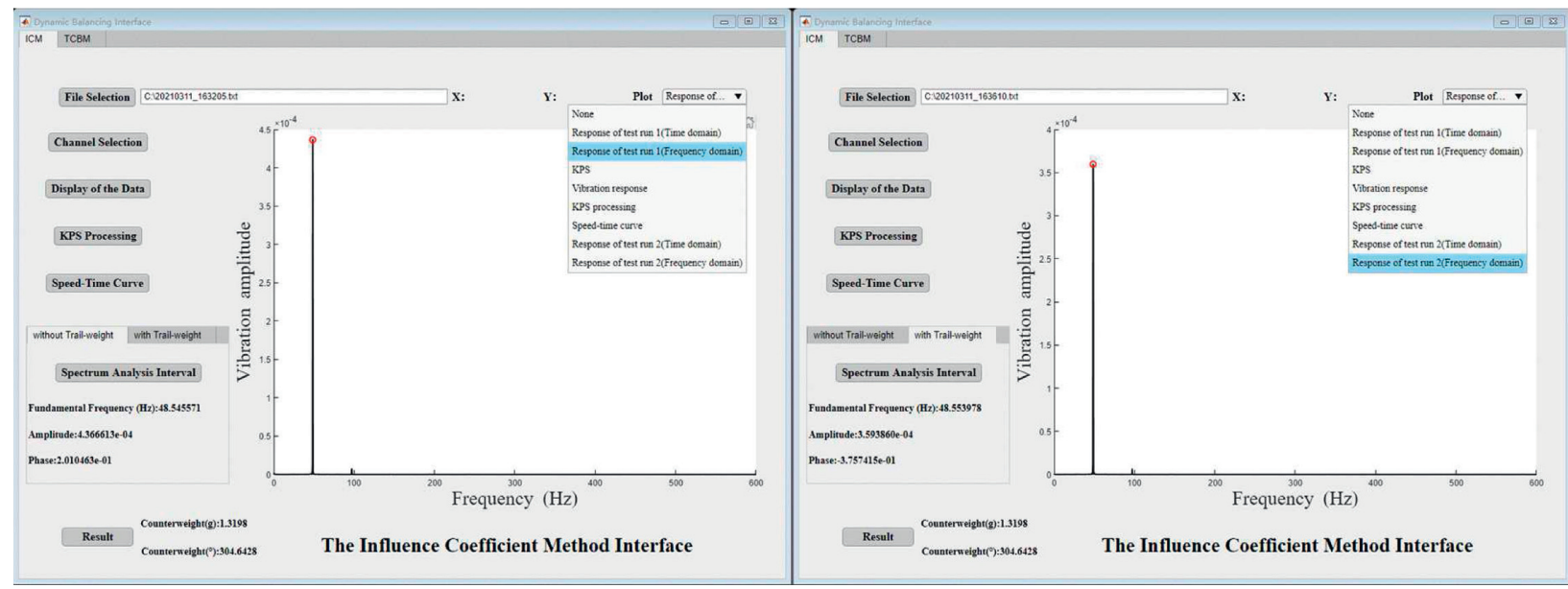

FIGURE 16: The counterweight identified by the ICM interface.

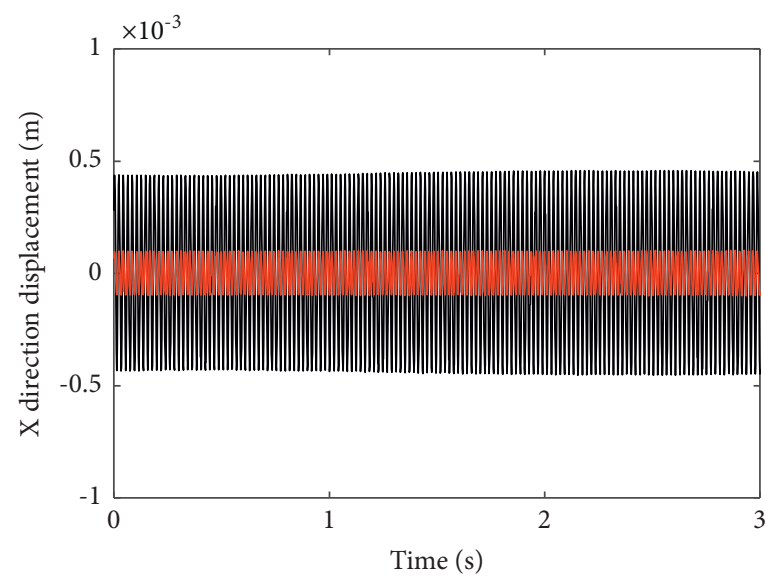

Before balancing

After balancing

FIgURE 17: Steady-state vibration response of the measuring point before and after balancing by the ICM interface. 


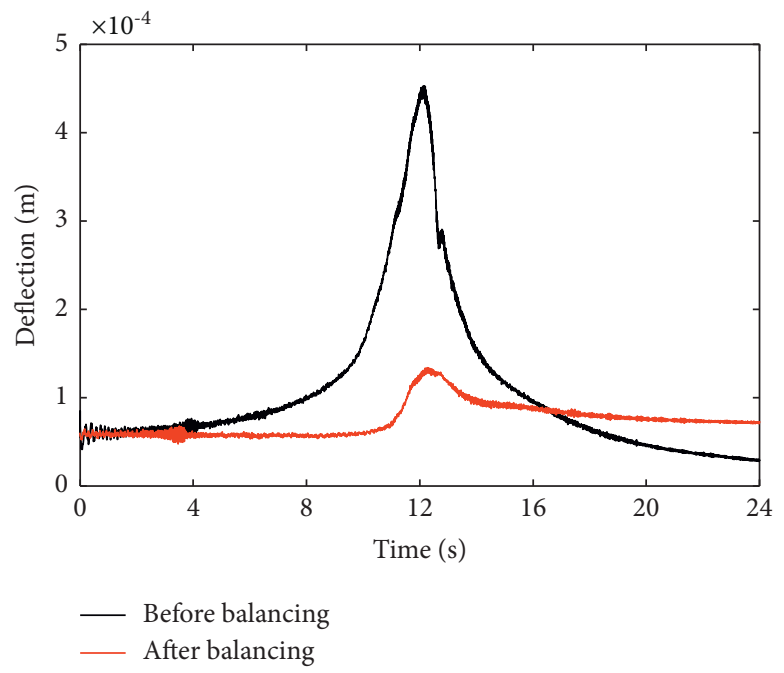

Figure 18: The transient deflection of the measuring point before and after balancing by the ICM interface.

$73.11 \%$ after balancing by the TCBM interface. Other than the TCBM interface, the ICM interface requires steady-state vibration response data while the rotor system should operate with constant rotational speed. In addition, the ICM interface requires two operations of the rotor system while one measures the signal of the rotor system without trail-weight and the other measures the signal of the rotor system with trail-weight. The steady-state vibration response amplitudes of $2914 \mathrm{r} / \mathrm{min}$ are significantly decreased after balancing by the ICM interface, and the maximum deflection of the measuring points has decreased by $70.00 \%$ after balancing by the ICM interface. The dynamic-balancing results calculated by the above two interfaces prove that the dynamic-balancing testing system can be effectively applied to the field of dynamic balancing of the flexible rotor system. And the testing system is more convenient than the source program of the dynamic-balancing methods in balancing the rotor system, simplifying the balancing steps and improving the balancing efficiency and safety.

\section{Conclusion}

In this paper, we designed a dynamic-balancing testing system for the flexible rotor system, whose innovative features are the interfaces according to the principles of TCBM and ICM for dynamic balancing of the rotor system. The functions of the testing system are monitoring the operations of the rotor synchronously, measuring and recording the required vibration response of the rotor, analyzing the dynamic characteristics of the rotor, and finally identifying the unbalance parameters of the rotor. The experiments of the single disc flexible rotor are carried out to detect the functions of the testing system and verify the effectiveness of the dynamic-balancing method. According to the experiment tests, some conclusions are made:

(1) The experimental tests of the dynamic-balancing testing system targeted to the single disc rotor system show that the hardware and the software can work well and realize their own respective functions.
(2) The maximum deflection of the measuring point has decreased by $73.11 \%$ after balancing by the TCBM interface. The maximum amplitude of the measuring point at $2914 \mathrm{r} / \mathrm{min}$ has decreased by $77.74 \%$ after balancing by ICM interface, while the maximum deflection during the whole operation has decreased by $70.00 \%$. The dynamic balancing results demonstrate the application of the above interfaces are both satisfactory for the field of dynamic balancing.

(3) In addition to the above good dynamic-balancing results, the testing system designed in this paper improves safety in balancing due to its function of monitoring the operations of the rotor synchronously. Meanwhile, the abstract source programs are integrated and designed in the form of buttons on the interface of the testing system. The functions of the source programs can be realized by clicking the corresponding buttons on the interface, which will make the balancing steps clearer. The functions of analyzing the measuring signals synchronously and processing the recording data on the interface make the testing system more efficient in signal processing.

In conclusion, the dynamic-balancing testing system could be successfully used in the field balancing of rotor system which has the advantages of simplifying the balancing process and improving the balancing efficiency.

\section{Data Availability}

The data used to support the findings of this study are available from the first author and the corresponding author upon request.

\section{Conflicts of Interest}

The authors declare no conflicts of interest. 


\section{Acknowledgments}

This study was funded by the National Natural Science Foundation of China (Grant nos. 12072263, 11972295, and 11802235), Natural Science Foundation of Shaanxi Province (Grant no. 2020JQ-129), and State Key Laboratory of Mechanical Behavior and System Safety of Traffic Engineering Structures (Grant no. KF2020-26).

\section{References}

[1] K. Lu, Y. Jin, Y. Chen et al., "Review for order reduction based on proper orthogonal decomposition and outlooks of applications in mechanical systems," Mechanical Systems and Signal Processing, vol. 123, 2019.

[2] C. Fu, Y. Xu, Y. Yang, K. Lu, F. Gu, and A. Ball, "Response analysis of an accelerating unbalanced rotating system with both random and interval variables," Journal of Sound and Vibration, vol. 466, 2020.

[3] Z. Xie and W. Zhu, "An investigation on the lubrication characteristics of floating ring bearing with consideration of multi-coupling factors," Mechanical Systems and Signal Processing, vol. 162, 2022.

[4] A. W. Lees and M. I. Friswell, "The evaluation of rotor imbalance in flexibly mounted machines," Journal of Sound and Vibration, vol. 208, no. 5, 1997.

[5] Y. Menshikov, "Identification of rotor unbalance as inverse problem of measurement," Advances in Pure Mathematics, vol. 3, no. 9, 2013.

[6] S. Zhong, L. Li, H. Chen, and Z. Lu, "A novel balancing method for rotor using unsupervised deep learning," Shock and Vibration, vol. 2021, Article ID 1800164, 9 pages, 2021.

[7] C. Li, H. She, Q. Tan, and B. Wen, "'The effect of blade vibration on the nonlinear characteristics of rotor-bearing system supported by nonlinear suspension'," Nonlinear Dynamics, vol. 89, no. 2, 2017.

[8] D. Zhang, J. Fu, Q. Zhang, and J. Hong, “An effective numerical method for calculating nonlinear dynamics of structures with dry friction: application to predict the vibration response of blades with underplatform dampers," Nonlinear Dynamics, vol. 88, no. 1, 2017.

[9] Y. Du, S. Zhou, X. Jing, Y. Peng, H. Wu, and N. Kwok, "Damage detection techniques for wind turbine blades: a review," Mechanical Systems and Signal Processing, vol. 141, 2020.

[10] F. Chu and Y. Tang, "Stability and non-linear responses of a rotor-bearing system with pedestal looseness," Journal of Sound and Vibration, vol. 241, no. 5, 2001.

[11] H. Ma, X. Zhao, Y. Teng, and B. Wen, "Analysis of dynamic characteristics for a rotor system with pedestal looseness," Shock and Vibration, vol. 18, no. 1-2, 2011.

[12] Y. Luo, B. Wu, W. Wang, and H. Hu, "Progress and prospecton research of pedestal looseness fault in the rotating machinery," Journal of Dalian National University, vol. 17, no. 5, 2015.

[13] P. Pennacchi, N. Bachschmid, and E. Tanzi, "Light and short arc rubs in rotating machines: experimental tests and modelling," Mechanical Systems and Signal Processing, vol. 23, no. 7, 2009.

[14] K. Prabith and I. R. P. Krishna, “The numerical modeling of rotor-stator rubbing in rotating machinery: a comprehensive review'," Nonlinear Dynamics, vol. 101, no. 2, 2020.

[15] H. Ma, C. Shi, Q. Han, and B. Wen, "Fixed-point rubbing fault characteristic analysis of a rotor system based on contact theory," Mechanical Systems and Signal Processing, vol. 38, no. $1,2013$.

[16] L. Hou, H. Chen, Y. Chen, K. Lu, and Z. Liu, "Bifurcation and stability analysis of a nonlinear rotor system subjected to constant excitation and rub-impact," Mechanical Systems and Signal Processing, vol. 125, 2019.

[17] A. K. Darpe, K. Gupta, and A. Chawla, "Transient response and breathing behaviour of a cracked jeffcott rotor," Journal of Sound and Vibration, vol. 272, no. 1-2, 2004.

[18] H. Ma, J. Zeng, R. Feng, X. Pang, Q. Wang, and B. Wen, "Review on dynamics of cracked gear systems," Engineering Failure Analysis, vol. 55, 2015.

[19] Y. Yang, Q. Wu, Y. Wang, W. Qin, and K. Lu, "Dynamic characteristics of cracked uncertain hollow-shaft," Mechanical Systems and Signal Processing, vol. 124, 2019.

[20] S. Xia, X. Zhang, Z. Liu, and S. Xu, "A survey of research on misalignment of rotary machinery," Journal of Vibration Measurement Diagnosis, vol. 18, no. 3, 1998.

[21] H. Zhang, X. Li, L. Jiang, D. Yang, and Y. Chen, "A review of misalignment of aero-engine rotor system," Hangkong Xuebao/Acta Aeronaut. Astronaut. Sin.vol. 40, no. 6, 2019.

[22] Q. Han, M. Wang, G. Zhao, and G. Feng, "A review of rotor system with misalignment," Journal of Dynamic Control, vol. 14, no. 1, 2016.

[23] T. P. Goodman, "A least-squares method for computing balance corrections," Journal of Engineering Industry, vol. 86, no. 3, 1964.

[24] J. W. Lund and J. Tonnesen, "Analysis and experiments on multi-plane," Journal of Engineering Industry, vol. 94, no. 1, 1972.

[25] R. E. D. Bishop and G. M. L. Gladwell, "The vibration and balancing of an unbalanced flexible rotor," Journal of Mechanical Engineering Science, vol. 1, no. 1, 1959.

[26] W. Kellenberger, "Should a flexible rotor Be balanced in N or $(\mathrm{N}+2)$ planes?" Journal of Mechanical Engineering Science, vol. 94, no. 2, 1972.

[27] M. B. Deepthikumar, A. S. Sekhar, and M. R. Srikanthan, "Modal balancing of flexible rotors with bow and distributed unbalance," Journal of Sound and Vibration, vol. 332, no. 24, 2013.

[28] J. Yao, L. Liu, F. Yang, Y. Su, F. Scarpa, and J. Gao, "Balancing optimization of a multiple speeds flexible rotor," Journal of Sound and Vibration, vol. 480, 2020.

[29] G. Bin, X. Li, J. Wu, and J. Gao, "Virtual dynamic balancing method without trial weights for multi-rotor series shafting based on finite element model analysis," Journal of Renewable and Sustainable Energy, vol. 6, no. 4, 2014.

[30] G. Bin, X. Li, Y. Shen, and J. Gao, "Whole-machine dynamic balancing method without trial weights for multi-span rotor shafting based on dynamic finite element model," Jixie Gongcheng Xuebao/Journal Mechanical Engineering, vol. 52, no. 21, 2016.

[31] X. Li, L. Zheng, and Z. Liu, "Balancing of flexible rotors without trial weights based on finite element modal analysis," Journal of Vibration and Control, vol. 19, no. 3, 2013.

[32] R. Ye, L. Wang, X. Hou, Z. Luo, and Q. Han, "Balancing method without trial weights for rotor systems based on similitude scale model," Frontiers of Mechanical Engineering, vol. 13, no. 4, 2018.

[33] D. Zou, H. Zhao, G. Liu, N. Ta, and Z. Rao, “Application of augmented Kalman filter to identify unbalance load of rotorbearing system: theory and experiment," Journal of Sound and Vibration, vol. 463, 2019. 
[34] S. Zhao, X. Ren, W. Deng, K. Lu, Y. Yang, and C. Fu, "A transient characteristic-based balancing method of rotor system without trail weights," Mechanical Systems and Signal Processing, vol. 148, 2021.

[35] P. Binetti, D. Trouchet, L. Pollini, M. Innocenti, T. Hamel, and F. Le Bras, "The flight control system of the Hovereye ${ }^{\circledR}$ VTOL UAV," in Proceedings of the Platform Innovations and System Integration for Unmanned Air, Land and Sea Vehicles (AVTSCI Joint Symposium), Brussels, Belgium, May 2007.

[36] T. Mikami and K. Uchiyama, "Design of flight control system for quad tilt-wing UAV," in Proceedings of the 2015 International Conference on Unmanned Aircraft Systems, ICUAS 2015, Denver, CO, USA, June 2015.

[37] C. Wang, F. Wang, L. Chen, and C. Zhang, "A system design for the testing platform of robot teleoperation with enhanced reality based on binocular vision," in Proceedings of the - 2009 International Forum on Information Technology and Applications, IFITA 2009, Chengdu, China, May 2009.

[38] F. Bian, R. Li, L. Zhao, Y. Liu, and P. Liang, "Interface design of a human-robot interaction system for dual-manipulators teleoperation based on virtual reality," in Proceedings of the 2018 IEEE International Conference on Information and Automation, ICIA 2018, Wuyishan, China, August 2018. 